\title{
INEQUALITIES FOR JACOBI POLYNOMIALS
}

\author{
UFFE HAAGERUP AND HENRIK SCHLICHTKRULL
}

\begin{abstract}
A Bernstein type inequality is obtained for the Jacobi polynomials $P_{n}^{(\alpha, \beta)}(x)$, which is uniform for all degrees $n \geq 0$, all real $\alpha, \beta \geq 0$, and all values $x \in[-1,1]$. It provides uniform bounds on a complete set of matrix coefficients for the irreducible representations of $\mathrm{SU}(2)$ with a decay of $d^{-1 / 4}$ in the dimension $d$ of the representation. Moreover it complements previous results of Krasikov on a conjecture of Erdélyi, Magnus and Nevai.
\end{abstract}

\section{INTRODUCTION}

For $\alpha, \beta \in \mathbb{R}, \alpha, \beta>-1$, and $n$ a non-negative integer we denote by $P_{n}^{(\alpha, \beta)}$ the Jacobi polynomial with the standard normalization. Recall that in terms of the Gauss hypergeometric function,

$$
P_{n}^{(\alpha, \beta)}(x)=\frac{\Gamma(n+\alpha+1)}{\Gamma(\alpha+1) \Gamma(n+1)}{ }_{2} F_{1}\left(-n, n+\alpha+\beta+1 ; \alpha+1 ; \frac{1-z}{2}\right) .
$$

Recall also that for a fixed pair $(\alpha, \beta)$ these functions are orthogonal polynomials on $[-1,1]$ for the weight function

$$
w^{(\alpha, \beta)}(x)=(1-x)^{\alpha}(1+x)^{\beta}
$$

with the explicit values

$$
\int_{-1}^{1} P_{n}^{(\alpha, \beta)}(x)^{2} w^{(\alpha, \beta)}(x) d x=\frac{2^{\alpha+\beta+1}}{2 n+\alpha+\beta+1} \frac{\Gamma(n+\alpha+1) \Gamma(n+\beta+1)}{\Gamma(n+1) \Gamma(n+\alpha+\beta+1)}
$$

(see [12, eq. (4.3.3)).

For $x \in[-1,1]$ and $\alpha, \beta \geq 0$ let

$$
g_{n}^{(\alpha, \beta)}(x)=\left(\frac{\Gamma(n+1) \Gamma(n+\alpha+\beta+1)}{\Gamma(n+\alpha+1) \Gamma(n+\beta+1)}\right)^{1 / 2}\left(\frac{1-x}{2}\right)^{\alpha / 2}\left(\frac{1+x}{2}\right)^{\beta / 2} P_{n}^{(\alpha, \beta)}(x),
$$

then these functions are orthogonal on $[-1,1]$ for the constant weight. Moreover

$$
\frac{1}{2} \int_{-1}^{1} g_{n}^{(\alpha, \beta)}(x)^{2} d x=\frac{1}{2 n+\alpha+\beta+1} .
$$

In suitable coordinates the functions $g_{n}^{(\alpha, \beta)}$ with arbitrary non-negative integers $\alpha, \beta$ and $n$ comprise a natural and complete set of matrix coefficients for the irreducible representations of $\mathrm{SU}(2)$ (see Section 2 below). The value $2 n+\alpha+\beta+1$ in (1) is exactly the dimension of the corresponding irreducible representation.

We shall prove the following uniform upper bound

Theorem 1.1. There exists a constant $C>0$ such that

$$
\left|\left(1-x^{2}\right)^{\frac{1}{4}} g_{n}^{(\alpha, \beta)}(x)\right| \leq C(2 n+\alpha+\beta+1)^{-\frac{1}{4}}
$$

for all $x \in[-1,1]$, all $\alpha, \beta \geq 0$ and all non-negative integers $n$.

Date: January 30, 2012.

2000 Mathematics Subject Classification. 22E46, 33C45. 
We have not made a serious effort to find the best value of $C$, but at least our proof shows that $C<12$.

With standard normalization the inequality in Theorem 1.1 amounts to the following uniform bound for the Jacobi polynomials

$$
\begin{aligned}
& (\sin \theta)^{\alpha+\frac{1}{2}}(\cos \theta)^{\beta+\frac{1}{2}}\left|P_{n}^{(\alpha, \beta)}(\cos 2 \theta)\right| \\
& \quad \leq \frac{C}{\sqrt{2}}(2 n+\alpha+\beta+1)^{-\frac{1}{4}}\left(\frac{\Gamma(n+\alpha+1) \Gamma(n+\beta+1)}{\Gamma(n+1) \Gamma(n+\alpha+\beta+1)}\right)^{1 / 2}
\end{aligned}
$$

for $0 \leq \theta \leq \pi / 2$. The decay rate of $1 / 4$ in Theorem 1.1 is optimal when $\alpha$ and $\beta$ tend to infinity, see Remark 4.4. However, if the pair $(\alpha, \beta)$ is fixed, then $P_{n}^{(\alpha, \beta)}(x)$ is $O\left(n^{-1 / 2}\right)$ for each $x \neq \pm 1$, cf. 12, Thm. 7.32.2. In particular, in Legendre's case $\alpha=\beta=0$ where $P_{n}^{(\alpha, \beta)}(x)$ specializes to the Legendre polynomial $P_{n}(x)$, the Bernstein inequality (refined by Antonov and Kholshevnikov)

$$
\left(1-x^{2}\right)^{1 / 4}\left|P_{n}(x)\right| \leq(4 / \pi)^{1 / 2}(2 n+1)^{-1 / 2}, \quad x \in[-1,1],
$$

is known to be sharp, see [12, Thm. 7.3.3, and [10]. We refer to [2] for a further discussion of the sharpest constant in (2), with a subset of the current parameter range.

It is of interest also to express our inequality in terms of the orthonormal polynomials defined by

$$
\hat{P}_{n}^{(\alpha, \beta)}(x)=\left(\frac{(2 n+\alpha+\beta+1) \Gamma(n+1) \Gamma(n+\alpha+\beta+1)}{2^{\alpha+\beta+1} \Gamma(n+\alpha+1) \Gamma(n+\beta+1)}\right)^{1 / 2} P_{n}^{(\alpha, \beta)}(x)
$$

for which

$$
\int_{-1}^{1} \hat{P}_{n}^{(\alpha, \beta)}(x)^{2} w^{(\alpha, \beta)}(x) d x=1 .
$$

Here our estimate reads

$$
\left(1-x^{2}\right)^{\frac{1}{4}} \sqrt{w^{\alpha, \beta}(x)}\left|\hat{P}_{n}^{(\alpha, \beta)}(x)\right| \leq \frac{C}{\sqrt{2}}(2 n+\alpha+\beta+1)^{\frac{1}{4}} .
$$

The following generalization of Bernstein's inequality (3) was conjectured by Erdélyi, Magnus and Nevai, 3],

$$
\left(1-x^{2}\right)^{\frac{1}{4}} \sqrt{w^{\alpha, \beta}(x)}\left|\hat{P}_{n}^{(\alpha, \beta)}(x)\right| \leq C^{\prime}(\alpha+\beta+2)^{1 / 4}
$$

for all $\alpha, \beta \geq-\frac{1}{2}$ and all integers $n \geq 0$, with a uniform constant $C^{\prime}>0$. A stronger form of the conjecture was recently established by Krasikov, 7], but only in the parameter range $\alpha, \beta \geq \frac{1+\sqrt{2}}{4}, n \geq 6$. Our estimate is valid for a more general range, but it involves $2 n+\alpha+\beta$ rather than $\alpha+\beta$. Note however that by combining our results with those of [7], one can remove Krasikov's restriction $n \geq 6$ in the parameter range for the validity of (4).

The estimate (2) implies a similar estimate for the ultrasperical (Gegenbauer) polynomials $C_{n}^{(\lambda)}(x)$, as these are directly related to the Jacobi polynomials $P_{n}^{(\alpha, \beta)}(x)$ with $\alpha=\beta=\lambda-\frac{1}{2}$. Previous to [7] this case had been considered in [8], and as above (2) allows the removal of a restriction on the degree.

The proof of Theorem 1.1 is based on an expression for $P_{n}^{(\alpha, \beta)}(x)$ as a contour integral, for which we can estimate the integrand by elementary analysis. The proof is simpler when $\alpha$ and $\beta$ are integers. In this case, which is treated in Section 3 , the contour is just a circle. The general case is the discussed in Section 5 . 


\section{Motivation From RePRESEnTAtion theORY}

It is well known that the irreducible representations of $\mathrm{SU}(2)$ can be expressed by Jacobi polynomials. In the physics literature it is customary to denote the corresponding matrix representations as Wigner's d-matrices. We recall a few details (see [14, $\S 38$, 13, Ch. 3, or [6]). The irreducible representations $\pi_{l}$ of $\mathrm{SU}(2)$ are parametrized by the non-negative integers or half-integers $l=0, \frac{1}{2}, 1, \ldots$, where $2 l+1$ is the corresponding dimension. The standard representation space for $\pi_{l}$ is the space $\mathcal{P}_{l}$ of polynomials in two complex variables $z_{1}, z_{2}$, homogeneous of degree $2 l$, on which the representation is given by

$$
\left[\pi_{l}\left(\begin{array}{ll}
a & b \\
c & d
\end{array}\right) f\right]\left(z_{1}, z_{2}\right)=f\left(a z_{1}+c z_{2}, b z_{1}+d z_{2}\right) .
$$

Let

$$
k_{\phi}=\left(\begin{array}{cc}
e^{i \phi} & 0 \\
0 & e^{-i \phi}
\end{array}\right) \quad \text { and } \quad t_{\theta}=\left(\begin{array}{cc}
\cos \theta & -\sin \theta \\
\sin \theta & \cos \theta
\end{array}\right)
$$

for $\phi, \theta \in \mathbb{R}$, then every element $A \in \mathrm{SU}(2)$ allows a decomposition of the form $A=k_{\phi} t_{\theta} k_{-\psi}$. The monomials $z_{1}^{j} z_{2}^{k}$ with $j+k=2 l$ form a basis for $\mathcal{P}_{l}$, and it is convenient to use the notation

$$
h_{p}^{l}\left(z_{1}, z_{2}\right)=z_{1}^{l-p} z_{2}^{l+p}
$$

where $p=-l,-l+1, \ldots, l$. Notice that these are weight vectors

$$
\pi_{l}\left(k_{\phi}\right) h_{p}^{l}=e^{-i 2 p \phi} h_{p}^{l}, \quad(p=-1, \ldots, l) .
$$

Choosing the inner product on $\mathcal{P}_{l}$ so that $\pi_{l}$ is unitary, the functions $h_{p}^{l}$ form an orthogonal basis. We denote by $\hat{h}_{p}^{l}$ the corresponding normalized basis vectors. For $A \in \mathrm{SU}(2)$ the matrix elements

$$
m_{p q}^{l}(A)=\left\langle\pi_{l}(A) \hat{h}_{q}^{l}, \hat{h}_{p}^{l}\right\rangle
$$

with $p, q=-l, \ldots, l$, form the so-called Wigner's d-matrix. Our result for the Jacobi polynomials implies the following.

Theorem 2.1. Let $C$ be the constant from Theorem 1.1, Then

$$
|\sin 2 \theta|^{1 / 2}\left|m_{p q}^{l}\left(k_{\phi} t_{\theta} k_{-\psi}\right)\right| \leq C(2 l+1)^{-1 / 4}
$$

for all $\phi, \theta, \psi \in \mathbb{R}$, all $l=0, \frac{1}{2}, 1, \ldots$ and all $p, q=-l, \ldots, l$. Moreover, the exponent $1 / 4$ on the right hand side is best possible.

Proof. Explicitly the matrix elements are given as follows (see [14, [13, 6]). For $p, q=-l, \ldots, l$ such that $|q| \leq p$,

$$
m_{p q}^{l}\left(k_{\phi} t_{\theta} k_{-\psi}\right)=e^{-i 2 p \phi} e^{i 2 q \psi} g_{n}^{(\alpha, \beta)}(\cos 2 \theta),
$$

where

$$
\alpha=p-q, \beta=p+q, n=l-p .
$$

For other values of $p$ and $q$ there are similar expressions, and in all cases one has

$$
\left|m_{p q}^{l}\left(k_{\phi} t_{\theta} k_{-\psi}\right)\right|=\left|g_{n}^{(\alpha, \beta)}(\cos 2 \theta)\right|
$$

where $\alpha=|p-q|, \beta=|p+q|$ and $n=l-\max \{|p|,|q|\}$. Moreover

$$
\operatorname{dim} \pi_{l}=2 l+1=2 n+\alpha+\beta+1 .
$$

Thus (5) follows directly from Theorem 1.1. For the last statement of Theorem 2.1, see Remark 4.4 
Remark 2.2. For $l$ integral $\pi_{l}$ descends to a representation of $\mathrm{SO}(3)$, and the matrix elements $m_{p 0}^{l}$ with $q=0$ descend to spherical harmonic functions on $S^{2} \simeq$ $\mathrm{SO}(3) / \mathrm{SO}(2)$. With the common normalization from quantum mechanics the spherical harmonics $Y_{l}^{m}$ with $-l \leq m \leq l$ satisfy

$$
Y_{l}^{m}(\theta, \phi)= \pm \frac{(2 l+1)^{1 / 2}}{(4 \pi)^{1 / 2}} g_{l-\alpha}^{(\alpha, \alpha)}(\cos \theta) e^{i m \phi},
$$

where $\alpha=|m|$. From Theorem 1.1 we obtain the uniform estimate

$$
|\sin \theta|^{1 / 2}\left|Y_{l}^{m}(\theta, \phi)\right| \leq \frac{C}{(4 \pi)^{1 / 2}}(2 l+1)^{1 / 4}
$$

for all $\theta, \phi$ and all integers $l, m$ with $|m| \leq l$.

The Jacobi polynomials are also related to the harmonic analysis on the complex spheres with respect to the action of the unitary group. The spherical functions for the pair $(U(q), U(q-1))$ are functions on the unit sphere in $\mathbb{C}^{q}$, and in suitable coordinates they can be expressed by means of Jacobi functions $P_{n}^{(\alpha, \beta)}$ with $\alpha=q-2$ (see [11, 5]). The direct motivation for the present paper was an application of this observation for $q=2$ to a study of $\operatorname{Sp}(2, \mathbb{R})$. In $[4$ the first author and de Laat apply the uniform estimates of the present paper for the case $\alpha=0$, to show that $\operatorname{Sp}(2, \mathbb{R})$ does not have the approximation property. Earlier, Bernstein's inequality (3) had been used in [9] with a similar purpose for the group $\operatorname{SL}(3, \mathbb{R})$.

\section{INTEGRAL PARAMETERS}

The proof is based on the following integral expression, which is obtained by applying Cauchy's formula to Rodrigues' formula for $P_{n}^{(\alpha, \beta)}(x)$ (see [12], eq. (4.3.1)),

$$
(1-x)^{\alpha}(1+x)^{\beta} P_{n}^{(\alpha, \beta)}(x)=\left(-\frac{1}{2}\right)^{n} I_{n}^{(\alpha, \beta)}(x)
$$

for $x \in(-1,1)$, where

$$
I_{n}^{(\alpha, \beta)}(x)=\frac{1}{2 \pi i} \int_{\gamma(x)} \frac{(1-z)^{n+\alpha}(1+z)^{n+\beta}}{(z-x)^{n}} \frac{d z}{z-x} .
$$

Here $\gamma(x)$ is any closed contour encircling $x$ in the positive direction. We assume in this section that $\alpha$ and $\beta$ are integers $\geq 0$. Without this assumption one would have to request also that $\gamma(x)$ does not enclose the points $z= \pm 1$. We shall take $\gamma(x)=C(x, r)$, the circle centered at $x$ and with a radius $r>0$ to be specified later.

The case $n=0$ will be treated separately in Lemma 4.3 below. Here we assume $n \geq 1$ and let $a=\alpha / n$ and $b=\beta / n$, then

$$
\begin{aligned}
I_{n}^{(\alpha, \beta)}(x) & =\frac{1}{2 \pi i} \int_{C(x, r)}\left(\frac{(1-z)^{a+1}(1+z)^{b+1}}{z-x}\right)^{n} \frac{d z}{z-x} \\
& =\frac{1}{2 \pi i} \int_{C(0, r)}\left(\frac{(1-x-s)^{a+1}(1+x+s)^{b+1}}{s}\right)^{n} \frac{d s}{s} .
\end{aligned}
$$

In order to select a suitable radius $r$ we look for the stationary points of the expression inside the parentheses, as a function of $s$. We let

$$
\psi(s)=(a+1) \log (1-x-s)+(b+1) \log (1+x+s)-\log s
$$

for $s \in \mathbb{C}$, and analyze the derivative

$$
\psi^{\prime}(s)=\frac{a+1}{s+x-1}+\frac{b+1}{s+x+1}-\frac{1}{s},
$$

which is independent of the branch cut used for the complex logarithm. Now

$$
\psi^{\prime}(s)=\frac{A s^{2}+B(x) s+C(x)}{(s+x-1)(x+s+1) s}
$$


where

$$
A=a+b+1, \quad B(x)=(a+b) x+a-b, \quad C(x)=1-x^{2} .
$$

The numerator is a second order polynomial in $s$ with the discriminant

$$
\begin{aligned}
\Delta(x) & =B(x)^{2}-4 A C(x) \\
& =(a+b+2)^{2} x^{2}+2\left(a^{2}-b^{2}\right) x+(a-b)^{2}-4(a+b+1),
\end{aligned}
$$

which coincides with the polynomial $\Delta$ defined in 1 . The polynomial $\Delta(x)$ has two real roots

$$
\left.\begin{array}{l}
x^{+} \\
x^{-}
\end{array}\right\}=\frac{b^{2}-a^{2} \pm 4 \sqrt{(a+1)(b+1)(a+b+1)}}{(a+b+2)^{2}}
$$

for which $-1 \leq x^{-}<x^{+} \leq 1$. For $x^{-}<x<x^{+}$we have $\Delta(x)<0$, and thus there are two conjugate solutions $s=s_{1}, s_{2}$ to the equation $A s^{2}+B(x) s+C(x)=0$. They are

$$
s_{1}, s_{2}=\frac{-B(x) \pm i \sqrt{-\Delta(x)}}{2 A} .
$$

Note that

$$
\left|s_{1}\right|^{2}=\left|s_{2}\right|^{2}=s_{1} s_{2}=\frac{C(x)}{A}=\frac{1-x^{2}}{a+b+1} .
$$

Hence, if we choose the radius

$$
r=\sqrt{\frac{1-x^{2}}{a+b+1}}
$$

then our contour $C(0, r)$ will pass through the stationary points of $\psi$. We define $r$ by (8) for all $x \in(-1,1)$ (also when $\Delta(x) \geq 0$ ).

We now find

$$
\left|I_{n}^{(\alpha, \beta)}(x)\right| \leq \frac{1}{2 \pi} \int_{0}^{2 \pi}\left|\left(1-x-r e^{i \theta}\right)^{1+a}\left(1+x+r e^{i \theta}\right)^{1+b} r^{-1}\right|^{n} d \theta,
$$

and write

$$
\left|\left(1-x-r e^{i \theta}\right)^{1+a}\left(1+x+r e^{i \theta}\right)^{1+b} r^{-1}\right|=e^{f(\cos \theta)}
$$

where

$$
\begin{aligned}
f(t)= & \frac{a+1}{2} \ln \left(r^{2}+(1-x)^{2}-2 r(1-x) t\right) \\
& +\frac{b+1}{2} \ln \left(r^{2}+(1+x)^{2}+2 r(1+x) t\right)-\ln (r)
\end{aligned}
$$

for $t \in[-1,1]$. Notice that we allow the possible value $f(t)=-\infty$ in the end points $t= \pm 1$. Let

$$
t_{2}=\frac{r^{2}+(1-x)^{2}}{2 r(1-x)}, \quad t_{1}=-\frac{r^{2}+(1+x)^{2}}{2 r(1+x)}
$$

then $t_{1} \leq-1$ and $1 \leq t_{2}$. It follows that

$$
f(t)=\frac{a+1}{2} \ln \left(t_{2}-t\right)+\frac{b+1}{2} \ln \left(t-t_{1}\right)+K
$$

where

$$
K=\frac{a+1}{2} \ln (1-x)+\frac{b+1}{2} \ln (1+x)+\frac{a+b}{2} \ln r+\frac{a+b+2}{2} \ln 2
$$

is independent of $t$. With (11) we can extend the domain of definition for $f$ to $\left[t_{1}, t_{2}\right] \supset[-1,1]$. For later reference we note that from (10) and (8) it follows that

$$
t_{1}=\frac{-(a+b+2)-(a+b) x}{2 \sqrt{a+b+1} \sqrt{1-x^{2}}}, \quad t_{2}=\frac{(a+b+2)-(a+b) x}{2 \sqrt{a+b+1} \sqrt{1-x^{2}}},
$$


and

$$
t_{2}-t_{1}=\frac{a+b+2}{\sqrt{a+b+1} \sqrt{1-x^{2}}}
$$

We have

$$
\left|I_{n}^{(\alpha, \beta)}(x)\right| \leq \frac{1}{2 \pi} \int_{0}^{2 \pi} e^{n f(\cos \theta)} d \theta
$$

From (11) we find

$$
f^{\prime}(t)=-\frac{a+1}{2\left(t_{2}-t\right)}+\frac{b+1}{2\left(t-t_{1}\right)}=\frac{(a+b+2)\left(t_{0}-t\right)}{2\left(t_{2}-t\right)\left(t-t_{1}\right)},
$$

where $t_{0}$ is the convex combination

$$
t_{0}=\frac{(a+1) t_{1}+(b+1) t_{2}}{a+b+2}=\frac{-a+b-(a+b) x}{2 \sqrt{a+b+1} \sqrt{1-x^{2}}} \in\left(t_{1}, t_{2}\right) .
$$

Moreover

$$
f^{\prime \prime}(t)=-\frac{a+1}{2\left(t_{2}-t\right)^{2}}-\frac{b+1}{2\left(t-t_{1}\right)^{2}}<0 .
$$

Hence the function $f(t)$ is concave and has a global maximum at $t_{0}$. We thus obtain the initial estimate

$$
\left|I_{n}^{(\alpha, \beta)}(x)\right| \leq \frac{1}{\pi} \int_{0}^{\pi} e^{n f(\cos \theta)} d \theta \leq e^{n f\left(t_{0}\right)} .
$$

Since

$$
t_{2}-t_{0}=\frac{(a+1)\left(t_{2}-t_{1}\right)}{a+b+2}, \quad t_{0}-t_{1}=\frac{(b+1)\left(t_{2}-t_{1}\right)}{a+b+2}
$$

we find

$$
f\left(t_{0}\right)=\frac{a+1}{2} \ln \frac{(a+1)\left(t_{2}-t_{1}\right)}{a+b+2}+\frac{b+1}{2} \ln \frac{(b+1)\left(t_{2}-t_{1}\right)}{a+b+2}+K,
$$

and from (12) and (14) it then follows that

$$
f\left(t_{0}\right)=\frac{1}{2} \ln \left(\frac{2^{a+b+2}(a+1)^{a+1}(b+1)^{b+1}}{(a+b+1)^{a+b+1}}(1-x)^{a}(1+x)^{b}\right) .
$$

Thus

$$
\begin{aligned}
e^{n f\left(t_{0}\right)} & \leq\left(\frac{2^{a+b+2}(a+1)^{a+1}(b+1)^{b+1}}{(a+b+1)^{a+b+1}}(1-x)^{a}(1+x)^{b}\right)^{n / 2} \\
& =\left(\frac{2^{a+b+2}(a+1)^{a+1}(b+1)^{b+1}}{(a+b+1)^{a+b+1}}\right)^{n / 2}(1-x)^{\alpha / 2}(1+x)^{\beta / 2} .
\end{aligned}
$$

The inequality

$$
\begin{aligned}
\frac{\Gamma(n+1) \Gamma(n+\alpha+\beta+1)}{\Gamma(n+\alpha+1) \Gamma(n+\beta+1)}( & \left.\frac{(a+1)^{a+1}(b+1)^{b+1}}{(a+b+1)^{a+b+1}}\right)^{n} \\
& \leq\left(\frac{(n+1)(n+\alpha+\beta+1)}{(n+\alpha+1)(n+\beta+1)}\right)^{1 / 2}
\end{aligned}
$$

will be shown in Lemma 4.1. Inserting (17) and (19) into our definition of $g_{n}^{(\alpha, \beta)}$ we obtain the initial bound

$$
\left|g_{n}^{(\alpha, \beta)}(x)\right| \leq\left(\frac{(n+1)(n+\alpha+\beta+1)}{(n+\alpha+1)(n+\beta+1)}\right)^{1 / 4} .
$$

In particular, since $(n+1)(n+\alpha+\beta+1) \leq(n+\alpha+1)(n+\beta+1)$ it follows that $\left|g_{n}^{(\alpha, \beta)}(x)\right| \leq 1$ (which could also be seen directly from the fact that $g_{n}^{(\alpha, \beta)}$ is a unitary matrix coefficient of orthonormal vectors). 
In order to improve the estimate we need to replace the inequality $f(t) \leq f\left(t_{0}\right)$ by a stronger inequality. In Proposition 3.1 below we shall establish the inequality

$$
f(t) \leq f\left(t_{0}\right)+\frac{D}{1+t_{0}^{2}} f^{\prime \prime}\left(t_{0}\right)\left(t-t_{0}\right)^{2}
$$

for $t \in[-1,1]$, with a suitable constant $D>0$. Following the argument from before and taking into account the second term in (21) we can then improve (17) with the extra factor

$$
\frac{1}{\pi} \int_{0}^{\pi} \exp \left(\frac{n D}{1+t_{0}^{2}} f^{\prime \prime}\left(t_{0}\right)\left(\cos \theta-t_{0}\right)^{2}\right) d \theta
$$

on the right hand side.

For the estimation of the exponential integral we use Lemma 3.6 below, which is applicable since $f^{\prime \prime}\left(t_{0}\right)<0$. We let

$$
u=t_{0} \sqrt{\frac{n D}{1+t_{0}^{2}}\left|f^{\prime \prime}\left(t_{0}\right)\right|}, \quad v=\sqrt{\frac{n D}{1+t_{0}^{2}}\left|f^{\prime \prime}\left(t_{0}\right)\right|},
$$

and observe that $u^{2}+v^{2}=n D\left|f^{\prime \prime}\left(t_{0}\right)\right|$. We thus obtain

$$
\left|I_{n}^{(\alpha, \beta)}(x)\right| \leq 2 e^{n f\left(t_{0}\right)}\left(n D\left|f^{\prime \prime}\left(t_{0}\right)\right|\right)^{-1 / 4}
$$

and hence (20) has been improved to

$$
\left|g_{n}^{(\alpha, \beta)}(x)\right| \leq\left(\frac{(n+1)(n+\alpha+\beta+1)}{(n+\alpha+1)(n+\beta+1)}\right)^{1 / 4} 2\left(n D\left|f^{\prime \prime}\left(t_{0}\right)\right|\right)^{-1 / 4} .
$$

From (15), (18) and (14) it follows that

$$
f^{\prime \prime}\left(t_{0}\right)=-\frac{a+b+2}{2\left(t_{0}-t_{1}\right)\left(t_{2}-t_{0}\right)}=-\frac{(a+b+1)(a+b+2)}{2(a+1)(b+1)}\left(1-x^{2}\right),
$$

and hence

$$
\left|f^{\prime \prime}\left(t_{0}\right)\right|=\frac{(\alpha+\beta+n)(\alpha+\beta+2 n)}{2(\alpha+n)(\beta+n)}\left(1-x^{2}\right) .
$$

Since

$$
\frac{n+\alpha+\beta+1}{(n+\alpha+1)(n+\beta+1)} \leq \frac{n+\alpha+\beta}{(n+\alpha)(n+\beta)}
$$

and

$$
\frac{n+1}{n(2 n+\alpha+\beta)} \leq \frac{3}{2 n+\alpha+\beta+1}
$$

for all $n \geq 1$ and $\alpha, \beta \geq 0$, it finally follows that

$$
\left|g_{n}^{(\alpha, \beta)}(x)\right| \leq C^{\prime}(\alpha+\beta+2 n+1)^{-1 / 4}\left(1-x^{2}\right)^{-1 / 4}
$$

where $C^{\prime}=2 \sqrt[4]{6 / D}=2 \sqrt[4]{168}<8$ with the value $D=1 / 28$ from below. This completes the proof of Theorem 1.1 in the integral case (up to the cited results from below).

Proposition 3.1. Fix $x \in[-1,1]$ and let $f(t)$ and $t_{0}$ be as above. Then

$$
f(t) \leq f\left(t_{0}\right)+\frac{1}{28\left(1+t_{0}^{2}\right)} f^{\prime \prime}\left(t_{0}\right)\left(t-t_{0}\right)^{2}
$$

for all $t \in[-1,1]$.

Proof. We begin the proof by a sequence of lemmas.

Lemma 3.2. The following relation holds

$$
(a+b)^{2}+4(a+b+1) t_{0}^{2}=\frac{2 a^{2}}{1-x}+\frac{2 b^{2}}{1+x} .
$$


Proof. Using (16) we obtain

$$
4(a+b+1) t_{0}^{2}=\frac{(a-b+(a+b) x)^{2}}{1-x^{2}} .
$$

On the other hand

$$
\frac{2 a^{2}}{1-x}+\frac{2 b^{2}}{1+x}=\frac{2\left(a^{2}+b^{2}+\left(a^{2}-b^{2}\right) x\right)}{1-x^{2}} .
$$

Hence (24) follows from the identity

$$
(a+b)^{2}\left(1-x^{2}\right)+(a-b+(a+b) x)^{2}=2\left(a^{2}+b^{2}+\left(a^{2}-b^{2}\right) x\right),
$$

which is straightforward.

Lemma 3.3. We have

$$
1-x^{2} \leq 16 \frac{(a+1)(b+1)}{(a+b+2)^{2}}\left(1+t_{0}^{2}\right)
$$

for all $x \in[-1,1]$.

Proof. Note first that if we replace the triple $(a, b, x)$ by $(b, a,-x)$, then $t_{1}, t_{0}, t_{2}$ are replaced by $-t_{2},-t_{0},-t_{1}$ and hence the asserted inequality is unchanged. We may thus assume that $a \leq b$.

It follows from Lemma 3.2 that

$$
(a+b)^{2}+4(a+b+1) t_{0}^{2} \geq \frac{2 b^{2}}{1+x}
$$

and therefore

$$
1+x \geq \frac{2 b^{2}}{(a+b)^{2}+4(a+b+1) t_{0}^{2}} .
$$

Hence

$$
1-x \leq 2-\frac{2 b^{2}}{(a+b)^{2}+4(a+b+1) t_{0}^{2}}=2 \frac{a^{2}+2 a b+4(a+b+1) t_{0}^{2}}{(a+b)^{2}+4(a+b+1) t_{0}^{2}} .
$$

and

$$
1-x^{2} \leq 2(1-x) \leq 4 \frac{a^{2}+2 a b+4(a+b+1) t_{0}^{2}}{(a+b)^{2}+4(a+b+1) t_{0}^{2}}
$$

Since the right hand side is an increasing function of $t_{0}^{2}$ we have for $t_{0}^{2} \leq 1$ that

$$
1-x^{2} \leq 4 \frac{a^{2}+2 a b+4(a+b+1)}{(a+b)^{2}+4(a+b+1)} \leq 16 \frac{(a+1)(b+1)}{(a+b+2)^{2}},
$$

where in the last step we used that $a \leq b$ implies $a^{2}+2 a b \leq 4 a b$. For $t_{0}^{2} \geq 1$ we obtain similarly

$$
1-x^{2} \leq 4 \frac{\left(a^{2}+2 a b\right) t_{0}^{2}+4(a+b+1) t_{0}^{2}}{(a+b)^{2}+4(a+b+1)} \leq 16 \frac{(a+1)(b+1)}{(a+b+2)^{2}} t_{0}^{2} .
$$

This completes the proof of Lemma 3.3

Lemma 3.4. We have

$$
t_{2}-t_{0} \geq \frac{1}{4\left(1+t_{0}^{2}\right)^{1 / 2}} \quad \text { and } \quad t_{0}-t_{1} \geq \frac{1}{4\left(1+t_{0}^{2}\right)^{1 / 2}} .
$$

Proof. It follows from (14) and Lemma 3.3 that

$$
t_{2}-t_{1} \geq \frac{(a+b+2)^{2}}{4 \sqrt{(a+1)(b+1)(a+b+1)}}\left(1+t_{0}^{2}\right)^{-1 / 2},
$$

and hence by (18)

$$
t_{2}-t_{0} \geq \frac{\sqrt{a+1}(a+b+2)}{4 \sqrt{(b+1)(a+b+1)}}\left(1+t_{0}^{2}\right)^{-1 / 2} .
$$


Using $(b+1)(a+b+1) \leq(a+b+2)^{2}$ and $\sqrt{a+1} \geq 1$ we obtain the first inequality in (25). The second one is analogous.

Lemma 3.5. We have

$$
\left(u-t_{1}\right)\left(t_{2}-u\right) \leq 14\left(1+t_{0}^{2}\right)\left(t_{0}-t_{1}\right)\left(t_{2}-t_{0}\right)
$$

for all $u \in\left[t_{1}, t_{2}\right]$ for which $-1 \leq u \leq t_{0}$ or $t_{0} \leq u \leq 1$.

Proof. We first assume $a \leq b$. Then by (18)

$$
u-t_{1} \leq t_{2}-t_{1}=\frac{a+b+2}{b+1}\left(t_{0}-t_{1}\right) \leq 2\left(t_{0}-t_{1}\right) .
$$

In order to estimate $t_{2}-u$ we first note that $\left|u-t_{0}\right| \leq 1+\left|t_{0}\right|$ and hence

$$
t_{2}-u \leq t_{2}-t_{0}+\left|t_{0}-u\right| \leq t_{2}-t_{0}+1+\left|t_{0}\right| \text {. }
$$

By Lemma 3.4

$$
1+\left|t_{0}\right| \leq \sqrt{2}\left(1+t_{0}^{2}\right)^{1 / 2} \leq 4 \sqrt{2}\left(1+t_{0}^{2}\right)\left(t_{2}-t_{0}\right)
$$

and hence

$$
t_{2}-u \leq(1+4 \sqrt{2})\left(1+t_{0}^{2}\right)\left(t_{2}-t_{0}\right) \leq 7\left(1+t_{0}^{2}\right)\left(t_{2}-t_{0}\right) .
$$

Now (27) and (28) together imply (26). The proof for $a \geq b$ is analogous.

We can now prove Proposition 3.1. Let $t \in[-1,1]$. It follows from (15), (26) and (23) that

$$
\begin{aligned}
\frac{f^{\prime}(u)}{u-t_{0}} & =-\frac{a+b+2}{2\left(u-t_{1}\right)\left(t_{2}-u\right)} \\
& \leq-\frac{a+b+2}{28\left(1+t_{0}^{2}\right)\left(t_{0}-t_{1}\right)\left(t_{2}-t_{0}\right)}=\frac{f^{\prime \prime}\left(t_{0}\right)}{14\left(1+t_{0}^{2}\right)}
\end{aligned}
$$

for all $u \in \mathbb{R}$ between $t$ and $t_{0}$. Hence

$$
\begin{aligned}
f(t) & =f\left(t_{0}\right)+\int_{t_{0}}^{t} f^{\prime}(u) d u \\
& \leq f\left(t_{0}\right)+\frac{f^{\prime \prime}\left(t_{0}\right)}{14\left(1+t_{0}^{2}\right)} \int_{t_{0}}^{t}\left(u-t_{0}\right) d u=f\left(t_{0}\right)+\frac{f^{\prime \prime}\left(t_{0}\right)}{28\left(1+t_{0}^{2}\right)}\left(t-t_{0}\right)^{2} .
\end{aligned}
$$

Lemma 3.6. Let $u, v \in \mathbb{R}$ with $u^{2}+v^{2}>0$. Then

$$
\frac{1}{\pi} \int_{0}^{\pi} e^{-(u+v \cos s)^{2}} d s \leq \frac{2}{\left(u^{2}+v^{2}\right)^{1 / 4}}
$$

Proof. We will show (29) with the slightly stronger bound

$$
\frac{\sqrt{2}}{\sqrt{\max \{|u|,|v|\}}} \text {. }
$$

The statement is invariant under the map $(u, v) \mapsto(-u,-v)$ and, using the substitution $s \mapsto \pi-s$, also under $v \mapsto-v$. Hence, it is sufficient to show

$$
\frac{1}{\pi} \int_{0}^{\pi} e^{-(u-v \cos s)^{2}} d s \leq \frac{\sqrt{2}}{\sqrt{\max \{u, v\}}}
$$

for $u \geq 0, v \geq 0$.

Suppose first $0 \leq u \leq v$, then $v \neq 0$. Let $\sigma \in\left[0, \frac{\pi}{2}\right]$ be such that $\cos \sigma=\frac{u}{v}$. Then

$$
u-v \cos s=v(\cos \sigma-\cos s)=2 v \sin \left(\frac{s+\sigma}{2}\right) \sin \left(\frac{s-\sigma}{2}\right) .
$$


Note that $\sin \left(\frac{s+\sigma}{2}\right) \geq\left|\sin \left(\frac{s-\sigma}{2}\right)\right|$ because $\sin ^{2}\left(\frac{s+\sigma}{2}\right)-\sin ^{2}\left(\frac{s-\sigma}{2}\right)=\sin s \sin \sigma \geq 0$ for $s \in[0, \pi]$ and $\sigma \in\left[0, \frac{\pi}{2}\right]$. Using also that $|\sin t| \geq \frac{2}{\pi}|t|$ for $|t| \leq \frac{\pi}{2}$, it follows that

$$
\begin{aligned}
\frac{1}{\pi} \int_{0}^{\pi} e^{-(u-v \cos s)^{2}} d s & =\frac{1}{\pi} \int_{0}^{\pi} e^{-4 v^{2} \sin ^{2}\left(\frac{s+\sigma}{2}\right) \sin ^{2}\left(\frac{s-\sigma}{2}\right)} d s \\
& \leq \frac{1}{\pi} \int_{0}^{\pi} e^{-4 v^{2} \pi^{-4}(s-\sigma)^{4}} d s \\
& \leq \frac{1}{\pi} \int_{-\infty}^{\infty} e^{-4 v^{2} \pi^{-4} s^{4}} d s \leq \frac{2}{\sqrt{2 v}}
\end{aligned}
$$

where we used that $\int_{0}^{\infty} e^{-t^{4}} d t=\Gamma\left(\frac{5}{4}\right) \leq 1$.

Suppose next $0 \leq v \leq u \leq 2 v$. Then $u-v \cos s \geq v(1-\cos s)=2 v \sin ^{2}\left(\frac{s}{2}\right)$. Hence,

$$
\begin{aligned}
\frac{1}{\pi} \int_{0}^{\pi} e^{-(u-v \cos s)^{2}} d s & \leq \frac{1}{\pi} \int_{0}^{\pi} e^{-4 v^{2} \sin ^{4}\left(\frac{s}{2}\right)} d s \\
& \leq \frac{1}{\pi} \int_{0}^{\pi} e^{-4 v^{2} \pi^{-4} s^{4}} d s \leq \frac{1}{\sqrt{2 v}} \leq \frac{1}{\sqrt{u}}
\end{aligned}
$$

using again $\int_{0}^{\infty} e^{-t^{4}} d t \leq 1$.

Suppose finally $0 \leq 2 v \leq u$. Then $u-v \cos s \geq \frac{u}{2}$ and hence

$$
\frac{1}{\pi} \int_{0}^{\pi} e^{-(u-v \cos s)^{2}} d s \leq e^{-\frac{u^{2}}{4}} \leq \frac{1}{\sqrt{u}}
$$

where we used that $x e^{-x^{4}} \leq \frac{1}{\sqrt{2}}$ for all $x \geq 0$.

\section{Some inequalities With Gamma FunCtions}

In this section we prove some inequalities which were used in the preceding section. We assume that $\alpha, \beta$ are real and non-negative.

Lemma 4.1. Let $n, \alpha, \beta \geq 0$. Then

$$
\begin{aligned}
& \frac{\Gamma(n+1) \Gamma(n+\alpha+\beta+1)}{\Gamma(n+\alpha+1) \Gamma(n+\beta+1)} \\
& \quad \leq \frac{n^{n}(\alpha+\beta+n)^{\alpha+\beta+n}}{(\alpha+n)^{\alpha+n}(\beta+n)^{\beta+n}}\left(\frac{(n+1)(n+\alpha+\beta+1)}{(n+\alpha+1)(n+\beta+1)}\right)^{1 / 2} .
\end{aligned}
$$

Proof. We have for $x, y, z \geq 0$

$$
\ln \frac{\Gamma(x+1) \Gamma(x+y+z+1)}{\Gamma(x+y+1) \Gamma(x+z+1)}=\int_{0}^{y} \int_{0}^{z}(\ln \Gamma)^{\prime \prime}(x+s+t+1) d t d s .
$$

We claim that

$$
(\ln \Gamma)^{\prime \prime}(u+1) \leq \frac{1}{u}-\frac{1}{2(u+1)^{2}}
$$

for all $u>0$. The asserted inequality (30) follows easily from (31) and (32).

In order to prove (32) we recall that

$$
(\ln \Gamma)^{\prime \prime}(u+1)=\sum_{k=1}^{\infty} \frac{1}{(u+k)^{2}}=\sum_{k=0}^{\infty} A(u+k),
$$

where

$$
A(u)=\frac{1}{(u+1)^{2}} .
$$

For the other side of (32) we use the telescoping series

$$
\frac{1}{u}=\sum_{k=0}^{\infty} B(u+k), \quad \frac{1}{2(u+1)^{2}}=\sum_{k=0}^{\infty} C(u+k),
$$


where

$$
B(u)=\frac{1}{u}-\frac{1}{u+1}=\frac{1}{u(u+1)}
$$

and

$$
C(u)=\frac{1}{2(u+1)^{2}}-\frac{1}{2(u+2)^{2}}=\frac{2 u+3}{2(u+1)^{2}(u+2)^{2}} .
$$

We observe that

$$
C(u) \leq \frac{1}{(u+1)^{2}(u+2)}
$$

and hence

$$
B(u)-C(u) \geq \frac{1}{u(u+1)}-\frac{1}{(u+1)^{2}(u+2)}=\frac{u^{2}+2 u+2}{u(u+1)^{2}(u+2)} \geq A(u) .
$$

We obtain (32) by termwise application of this inequality to the series.

Lemma 4.2. For $\alpha, \beta \geq 0$

$$
\frac{\Gamma(\alpha+\beta+1)}{\Gamma(\alpha+1) \Gamma(\beta+1)} \leq \frac{\left(\alpha+\beta+\frac{1}{2}\right)^{\alpha+\beta+\frac{1}{2}}\left(\frac{1}{2}\right)^{\frac{1}{2}}}{\left(\alpha+\frac{1}{2}\right)^{\alpha+\frac{1}{2}}\left(\beta+\frac{1}{2}\right)^{\beta+\frac{1}{2}}} .
$$

Proof. Following the preceding proof one deduces this inequality from

$$
(\ln \Gamma)^{\prime \prime}(u+1) \leq \frac{1}{u+\frac{1}{2}}
$$

The latter inequality is also seen as in the preceding proof, by using the telescoping series

$$
\frac{1}{u+\frac{1}{2}}=\sum_{k=0}^{\infty} D(u+k)
$$

where

$$
D(u)=\frac{1}{u+\frac{1}{2}}-\frac{1}{u+\frac{3}{2}}=\frac{1}{\left(u+\frac{1}{2}\right)\left(u+\frac{3}{2}\right)} \geq \frac{1}{(u+1)^{2}}=A(u) .
$$

Lemma 4.3. Let $\alpha, \beta \geq 0$ and $-1 \leq x \leq 1$. Then

$$
0 \leq\left(1-x^{2}\right)^{1 / 4} g_{0}^{(\alpha, \beta)}(x) \leq(\alpha+\beta+1)^{-1 / 4} .
$$

Proof. Since $P_{0}^{(\alpha, \beta)}(x)=1$, we have $g_{0}^{(\alpha, \beta)}(x) \geq 0$ and

$$
\left(1-x^{2}\right)^{\frac{1}{2}} g_{0}^{(\alpha, \beta)}(x)^{2}=\frac{2 \Gamma(\alpha+\beta+1)}{\Gamma(\alpha+1) \Gamma(\beta+1)}\left(\frac{1-x}{2}\right)^{\alpha+\frac{1}{2}}\left(\frac{1+x}{2}\right)^{\beta+\frac{1}{2}} .
$$

For $\mu, \nu \geq 0$ the function $\varphi(x)=(1-x)^{\mu}(1+x)^{\nu}$ on $[-1,1]$ satisfies

$$
\max _{x \in[-1,1]} \varphi(x)=\varphi\left(\frac{\nu-\mu}{\nu+\mu}\right)=\frac{2^{\mu+\nu} \mu^{\mu} \nu^{\nu}}{(\mu+\nu)^{\mu+\nu}} .
$$

Hence by Lemma 4.2

$$
\begin{aligned}
\max _{x \in[-1,1]}\left(1-x^{2}\right)^{\frac{1}{2}} g_{0}^{(\alpha, \beta)}(x)^{2} & =\frac{2 \Gamma(\alpha+\beta+1)}{\Gamma(\alpha+1) \Gamma(\beta+1)} \frac{\left(\alpha+\frac{1}{2}\right)^{\alpha+\frac{1}{2}}\left(\beta+\frac{1}{2}\right)^{\beta+\frac{1}{2}}}{(\alpha+\beta+1)^{\alpha+\beta+1}} \\
& \leq h(\alpha+\beta)(\alpha+\beta+1)^{-1 / 2}
\end{aligned}
$$

where

Since

$$
h(t)=\sqrt{2}\left(\frac{t+\frac{1}{2}}{t+1}\right)^{t+\frac{1}{2}} .
$$

$$
(\log h)^{\prime}(t)=\frac{1}{2(t+1)}+\log \left(\frac{t+\frac{1}{2}}{t+1}\right)=\int_{t+\frac{1}{2}}^{t+1}\left(\frac{1}{t+1}-\frac{1}{u}\right) d u \leq 0
$$


it follows that $h(t) \leq h(0)=1$ for all $t \geq 0$. This proves lemma 4.3

Remark 4.4. It follows from (33) and Stirling's formula that

$$
\max \left(1-x^{2}\right)^{1 / 4}\left|g_{0}^{(\alpha, \beta)}(x)\right| \sim(2 / \pi)^{1 / 4}(\alpha+\beta+1)^{-1 / 4}
$$

when $\alpha \rightarrow \infty$ and $\beta \rightarrow \infty$. Hence the decay rate $1 / 4$ in Theorem 1.1 cannot be improved. This was observed already in [3], p. 604.

In this connection it can be noted that for each $l=0, \frac{1}{2}, 1, \ldots$, the irreducible representation $\pi_{l}$ of $\mathrm{SU}(2)$ will exhibit matrix coefficients in which the functions $g_{0}^{(\alpha, \beta)}$ for $\alpha+\beta=2 l$ occur (see Section Q). In particular, it follows that a positive solution to the EMN-conjecture mentioned in the introduction, will not significantly improve the representation theoretic content of Theorem 1.1. discussed in Section 2 .

\section{The General CASE}

In this section $n \in \mathbb{N}_{0}$ and $\alpha, \beta$ are non-negative real numbers. We have already proved in Lemma 4.3 that

$$
\left|g_{0}^{(\alpha, \beta)}(x)\right| \leq(\alpha+\beta+1)^{-1 / 4}, \quad x \in[-1,1], \alpha, \beta \geq 0,
$$

so we can assume that $n>0$. As in Section [3 we put $a=\alpha / n$ and $b=\beta / n$ and use the integral representation (6) -(17) of $P_{n}^{(\alpha, \beta)}(x)$, with a closed contour $\gamma(x)$ encircling $x$ in the positive direction. In addition we assume now that $\gamma(x)$ does not intersect the branch cuts ] $-\infty,-1]$ and $[1, \infty[$. As before we define $r>0$ by (8) and consider the circle $C(x, r)$. For $|x|<1$ we find

$$
1<x+r \quad \Leftrightarrow \quad x>\frac{a+b}{a+b+2},
$$

and consequently

$$
-1>x-r \quad \Leftrightarrow \quad x<-\frac{a+b}{a+b+2} .
$$

Hence we can distinguish the following cases:

Case 1: $\frac{a+b}{a+b+2}<x<1$. Then 1 is inside and -1 is outside $C(x, r)$.

Case 2: $|x|<\frac{a+b}{a+b+2}$. Both 1 and -1 are outside $C(x, r)$.

Case 3: $-1<x<-\frac{a+b}{a+b+2}$. Here 1 is outside and -1 is inside $C(x, r)$.

By continuity it suffices to prove Theorem 1.1 in each of these three cases. As the proof given in Section 3 is valid without modification in Case 2, we need only consider the other two cases. Note that the integral

$$
J_{n}^{(\alpha, \beta)}(x):=\frac{1}{2 \pi i} \int_{C(x, r)} \frac{(1-z)^{n+\alpha}(1+z)^{n+\beta}}{(z-x)^{n+1}} d z
$$

makes sense for all $\alpha, \beta \geq 0$, although the argument of the integrand may become discontinuous at $z=x+r$ or at $z=x-r$ when these points belong to the branch cuts. As in Section 3, see (17),

$$
\left|J_{n}^{(\alpha, \beta)}(x)\right| \leq \frac{1}{\pi} \int_{0}^{\pi} e^{n f(\cos \theta)} d \theta
$$

where $f$ is the function defined by (9). Note that $f$ depends on $a, b$ and $x$. When necessary we denote it by $f=f_{a, b, x}$.

Lemma 5.1. The integral (7) satisfies

$$
I_{n}^{(\alpha, \beta)}(x)=J_{n}^{(\alpha, \beta)}(x)+R_{n}^{(\alpha, \beta)}(x)
$$

where $\left|R_{n}^{(\alpha, \beta)}(x)\right| \leq e^{n f(1)}$ in Case 1, $R_{n}^{(\alpha, \beta)}(x)=0$ in Case 2, and $\left|R_{n}^{(\alpha, \beta)}(x)\right| \leq$ $e^{n f(-1)}$ in Case 3 . 
Proof. Consider first Case 1, and note that

$$
f(1)=\ln \left((r-1+x)^{a+1}(r+1+x)^{b+1} r^{-1}\right) .
$$

We let the closed contour $\gamma(x)$ follow $C(x, r)$ except for a small arc around the possible locus of discontinuity at $x+r$. Let $\delta>0$ be such that the removed arc consist of points $z_{1}+i z_{2}$ in the strip $\left|z_{2}\right|<\delta$. The end points below and above $x+r$ are joined to $1 \pm i \delta$ by line segments along the axis. Finally $1-i \delta$ and $1+i \delta$ are connected by a half circle crossing the axis to the left of 1 . In the limit $\delta \rightarrow 0^{+}$ we obtain (34) with

$$
\begin{aligned}
R_{n}^{(\alpha, \beta)}(x) & =-\frac{\sin (\pi(n+\alpha))}{\pi} \int_{1}^{x+r} \frac{(z-1)^{n+\alpha}(1+z)^{n+\beta}}{(z-x)^{n+1}} d z \\
& =(-1)^{n-1} \frac{\sin (\pi \alpha)}{\pi} \int_{1-x}^{r} \frac{(s+x-1)^{n+\alpha}(1+s+x)^{n+\beta}}{s^{n+1}} d s .
\end{aligned}
$$

In particular, $R_{n}^{(\alpha, \beta)}(x)=0$ if $\alpha=0$ so that we may assume $\alpha>0$. For $x<1$ and $0<s<r$ we have $\frac{s}{r}(1-x) \leq 1-x$ and hence $s+x-1 \leq \frac{s}{r}(r+x-1)$. It follows that

$$
\frac{(s+x-1)^{n+\alpha}(1+s+x)^{n+\beta}}{s^{n+1}} \leq \frac{(r+x-1)^{n+\alpha}(1+r+x)^{n+\beta} s^{\alpha-1}}{r^{n+\alpha}}
$$

for $0<1-x<s<r$. Thus

$$
\begin{aligned}
\left|R_{n}^{(\alpha, \beta)}(x)\right| & \leq \frac{|\sin (\pi \alpha)|}{\pi} \frac{(r+x-1)^{n+\alpha}(1+r+x)^{n+\beta}}{r^{n+\alpha}} \int_{0}^{r} s^{\alpha-1} d s \\
& =\frac{|\sin (\pi \alpha)|}{\pi \alpha} \frac{(r+x-1)^{n+\alpha}(1+r+x)^{n+\beta}}{r^{n}}=\frac{|\sin (\pi \alpha)|}{\pi \alpha} e^{n f(1)}
\end{aligned}
$$

completing the proof for Case 1.

Case 2 is trivial since 1 and -1 are both outside $C(x, r)$. For the last case we observe that

and likewise

$$
I_{n}^{(\alpha, \beta)}(x)=(-1)^{n} I_{n}^{(\beta, \alpha)}(-x)
$$

$$
J_{n}^{(\alpha, \beta)}(x)=(-1)^{n} J_{n}^{(\beta, \alpha)}(-x) .
$$

Moreover, from (9) we see that $f_{b, a,-x}(t)=f_{a, b, x}(-t)$. Now Case 3 follows easily from Case 1.

Lemma 5.2. Let $t_{0} \in\left(t_{1}, t_{2}\right)$ be given by (16). Then

$$
f(1) \leq f\left(t_{0}\right)+\frac{1}{140} f^{\prime \prime}\left(t_{0}\right)
$$

in Case 1, and likewise, in Case 3,

$$
f(-1) \leq f\left(t_{0}\right)+\frac{1}{140} f^{\prime \prime}\left(t_{0}\right) .
$$

Proof. It follows from (16) that the derivative of $t_{0}=t_{0}(x)$ as a function of $x$ is

$$
\frac{-(a+b)+(b-a) x}{2(a+b+1)^{1 / 2}\left(1-x^{2}\right)^{3 / 2}} .
$$

Since $|b-a| \leq a+b$ it follows that $t_{0}$ is a decreasing function of $x \in(-1,1)$. Hence in Case 1,

$$
t_{0}(x)<t_{0}\left(\frac{a+b}{a+b+2}\right)=\frac{(b-a)(a+b+2)-(a+b)^{2}}{4(a+b+1)} \leq \frac{1}{2}
$$

where the last inequality follows from

$$
(b-a)(a+b+2)-(a+b)^{2}=-2 a(a+b+1)+2 b \leq 2(a+b+1) .
$$


From Proposition 3.1 and (23) we have

$$
f(1) \leq f\left(t_{0}\right)+\frac{\left(1-t_{0}\right)^{2}}{28\left(1+t_{0}\right)^{2}} f^{\prime \prime}\left(t_{0}\right)
$$

with $f^{\prime \prime}\left(t_{0}\right)<0$. Since $t_{0} \leq \frac{1}{2}$ we find

$$
4 t_{0}^{2}-10 t_{0}+4=4\left(t_{0}-\frac{1}{2}\right)\left(t_{0}-2\right) \geq 0
$$

and

Hence

$$
\frac{\left(1-t_{0}\right)^{2}}{1+t_{0}^{2}}-\frac{1}{5}=\frac{4 t_{0}^{2}-10 t_{0}+4}{5\left(1+t_{0}^{2}\right)} \geq 0
$$

$$
f(1) \leq f\left(t_{0}\right)+\frac{1}{140} f^{\prime \prime}\left(t_{0}\right)
$$

as claimed. The proof in Case 3 follows by the observation at the end of the proof of Lemma 5.1. since the $t_{0}$ associated with the data $b, a,-x$ is the negative of the $t_{0}$ associated with $a, b, x$.

We can now complete the proof of Theorem 1.1. As in (22) we find

$$
\left|J_{n}^{(\alpha, \beta)}(x)\right| \leq \frac{1}{\pi} \int_{0}^{\pi} e^{n f(\cos \theta)} d \theta \leq C_{1} e^{n f\left(t_{0}\right)}\left(n\left|f^{\prime \prime}\left(t_{0}\right)\right|\right)^{-1 / 4}
$$

where $C_{1}=2 D^{-1 / 4}=2 \sqrt[4]{28}$. Since $e^{-t} \leq \frac{1}{\sqrt{2}} t^{-1 / 4}$ for all $t>0$ we obtain from Lemmas 5.1 and 5.2 that

$$
\left|R_{n}^{(\alpha, \beta)}(x)\right| \leq C_{2} e^{n f\left(t_{0}\right)}\left(n\left|f^{\prime \prime}\left(t_{0}\right)\right|\right)^{-1 / 4}
$$

with $C_{2}=\frac{1}{\sqrt{2}} \sqrt[4]{140}=\sqrt[4]{35}$. All together

$$
\left|I_{n}^{(\alpha, \beta)}(x)\right| \leq C_{3} e^{n f\left(t_{0}\right)}\left(n\left|f^{\prime \prime}\left(t_{0}\right)\right|\right)^{-1 / 4}
$$

with $C_{3}=C_{1}+C_{2}$. Still proceeding as in Section 3 and using Lemma 4.1] we finally get

$$
\begin{aligned}
\left|g_{n}^{(\alpha, \beta)}(x)\right| & \leq C_{3}\left(\frac{(n+1)(n+\alpha+\beta+1)}{(n+\alpha+1)(n+\beta+1)}\right)^{1 / 4}\left(n\left|f^{\prime \prime}\left(t_{0}\right)\right|\right)^{-1 / 4} \\
& \leq C(1+\alpha+\beta+2 n)^{-1 / 4}\left(1-x^{2}\right)^{-1 / 4}
\end{aligned}
$$

for $C=\sqrt[4]{6} C_{3}$. In particular, we find $C<12$.

\section{REFERENCES}

[1] L. Chen and M. Ismail, On asymptotics of Jacobi polynomials. SIAM J. Math. Anal. 22 (1991), 1442-1449.

[2] W. Gautschi, How sharp is Bernstein's inequality for Jacobi polynomials? Electron. Trans. Numer. Anal. 36 (2009/10), 1-8.

[3] T. Erdélyi, A. P. Magnus, and P. Nevai, Generalized Jacobi weights, Christoffel functions, and Jacobi polynomials. SIAM J. Math. Anal. 25 (1994), 602-614.

[4] U. Haagerup and T. de Laat, Simple Lie groups without the approximation property. Preprint (2012), arXiv: 1201.1250.

[5] T. H. Koornwinder, The addition formula for Jacobi polynomials II. The Laplace type integral representation and the product formula. Mathematical Centre, Amsterdam 1976.

[6] T. H. Koornwinder, Representations of SU(2) and Jacobi polynomials. Unpublished lecture notes, 2008.

[7] I. Krasikov, An upper bound on Jacobi polynomials, J. Approx. Theory 149 (2007), 116-130.

[8] I. Krasikov, On the Erdélyi-Magnus-Nevai conjecture for Jacobi polynomials, Constr. Approx. 28 (2008), 113-125.

[9] V. Lafforgue and M. De la Salle, Noncommutative Lp-spaces without the completely bounded approximation property, Duke Math. J. 160 (2011), 71-116.

[10] L. Lorch, Alternative proof of a sharpened form of Bernstein's inequality for Legendre polynomials, Applicable Anal. 14 (1982/83), 237-240. 
[11] R. Sapiro, Special functions related to representations of the group $\mathrm{SU}(n)$ of class I relative to $\mathrm{SU}(n-1)$. Izv. Vyš̌. Učebn. Zaved. Matematika 1968 (1968), 97-107 (russian). English translation in Amer. Math. Soc. Translations, Series 2, 113 (1979), 201-211.

[12] G. Szegö, Orthogonal Polynomials. Amer. Math. Soc., 1939.

[13] N. Vilenkin, Special functions and the theory of group representations. Amer. Math. Soc., 1968.

[14] D. P. Zelobenko, Compact Lie groups and their representations. Amer. Math. Soc., 1973.

Department of Mathematical Sciences, University of Copenhagen, Denmark

E-mail address: haagerup@math.ku.dk, schlicht@math.ku.dk 